\title{
Operação de uma planta piloto na avaliação do desempenho de uma bomba centrífuga.
}

\author{
Juan Nicolas Andrade Cavalcante* (Graduando em Engenharia Química da Universidade Federal de \\ Campina Grande - UFCG); \\ Carla Gabriela Azevedo Misael (Graduanda em Engenharia Química da Universidade Federal de \\ Campina Grande - UFCG); \\ Caroline Vasconcelos Fernandes (Graduanda em Engenharia Química da Universidade Federal de \\ Campina Grande - UFCG); \\ Filipe J osé Ferreira Chaves (Graduando em Engenharia Química da Universidade Federal de Campina

$$
\text { Grande - UFCG); }
$$ \\ J osiele Souza Batista Santos (Graduanda em Engenharia Química da Universidade Federal de Campina \\ Grande - UFCG); \\ Suênia Fernandes de Vasconcelos (Graduanda em Engenharia Química da Universidade Federal de \\ Campina Grande - UFCG). \\ *E-mail: nicolas.engq@gmail.com
}

resumo:

Devido a grande necessidade de sistema de bombeamento em indústrias e até mesmo no cotidiano da sociedade, tornou-se fundamental o estudo aprofundado sobre o comportamento das variáveis relacionadas a cada tipo de bomba, visto que essas variáveis quando comparadas com as variáveis do próprio sistema em questão pode-se determinar se ocorrerá o fenômeno de cavitação da bomba ou não. Para a análise de determinada bomba em um requerido sistema, é essencial a avaliação das curvas características que descrevem o comportamento operacional das bombas. Nesse contexto, este trabalho tem como objetivo avaliar o desempenho de uma bomba centrífuga com duas velocidades de rotação, através das curvas características realizadas a partir das variáveis comportamentais altura de projeto, potência da bomba e eficiência da bomba, que foi encontrada a partir do tubo de Venturi. Para tal finalidade o estudo propõe uma planta piloto que reproduz um sistema de bombeamento, onde o fluido recorrente no sistema foi a água. Através da análise dos dados obtidos percebeu-se a proporcionalidade entre as variáveis altura de projeto, vazão e velocidade, a eficiência da bomba manteve seu comportamento similar em todas as etapas mostrando sua independência em relação a velocidade.

pallavras-chave:

Bomba centrífuga; Altura de projeto; Potência; Eficiência. 


\section{I ntrodução}

O transporte de produtos através de bombeamento em processos industriais é uma operação unitária constantemente necessária nas diversas fases do processamento. As necessidades de transportar de um nível a outro mais elevado ou mesmo alimentar um equipamento ou tanques de mistura que se encontram sob pressão mais elevada que o ambiente, é normalmente realizado por bombas (SOUSA, 2009).

As bombas são máquinas operatrizes hidráulicas que conferem energia ao fluído com a finalidade de transportá-lo de um ponto para outro obedecendo às condições de processo (DE FALCO \& MATOS, 2005). Esses equipamentos recebem energia de uma fonte motora qualquer e cedem parte ao fluido, majoritariamente sob forma de energia de pressão e cinética.

Na indústria do petróleo, a importância desses equipamentos é enorme. Depois dos motores elétricos, as bombas são os equipamentos rotativos que existem em maior quantidade em uma unidade industrial. O campo de aplicação das bombas é vasto. Em uma plataforma de petróleo, os principais serviços envolvendo bombas são: injeção de água, exportação de óleo, transferência de óleo, captação de água do mar e combate ao incêndio. Entretanto, existem mais dezenas de aplicações, como por exemplo, sistema de esgoto, produção de água doce etc (BONNIEARD, 2011).

As tubulações dos sistemas de bombeamento oferecem resistência ao escoamento do fluido. Parte da energia fornecida pela bomba ao fluido é perdida na resistência oferecida pelas tubulações ao escoamento. O motor da bomba, a bomba e o sistema de tubulações possuem um rendimento para cada ponto de operação do sistema. A eficiência energética de um sistema de bombeamento depende do rendimento destes três componentes (BRIDI, 2013)

Os custos atuais da energia elétrica não permitem mais que se trabalhe com equipamentos de baixo rendimento. Quando se trabalha com equipamentos de baixo rendimento, uma grande quantidade de energia é desperdiçada. Financeiramente, isso resulta numa redução dos lucros sobre os produtos industrializados ou sobre os serviços prestados. Bons lucros são obtidos reduzindo-se ao mínimo os desperdícios (BRIDI, 2013).

Nesse contexto este trabalho tem como finalidade analisar o comportamento das variáveis altura de projeto, potência da bomba e eficiência da bomba através das curvas características do sistema, além de observar a dependência e proporcionalidade entre as variáveis e o desempenho da bomba centrífuga em estudo.

\section{Metodologia}

Esse experimento foi realizado no Laboratório de Engenharia Química III da Unidade Acadêmica de Engenharia Química da Universidade Federal de Campina Grande.

Inicialmente, ligou-se o sistema de bombeamento, Figura 1. Em seguida, fixou-se uma velocidade e variou-se o $\Delta \mathrm{P}$ venturi com valores entre 0,1 a 0,5 . 


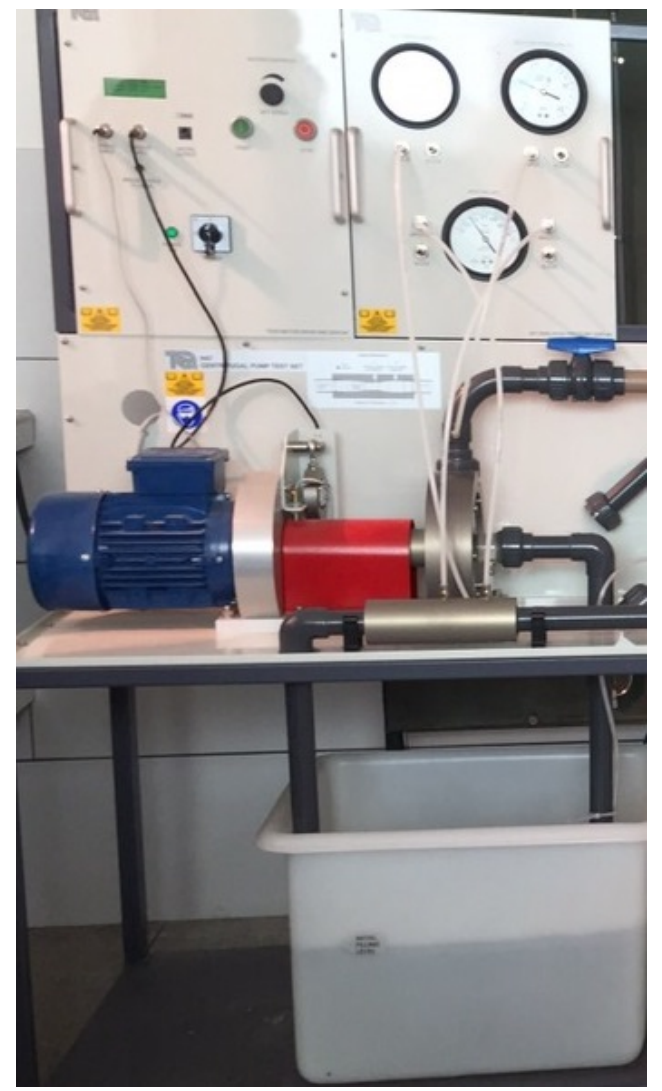

Figura 1 - Sistema de bombeamento utilizado na realização do experimento.

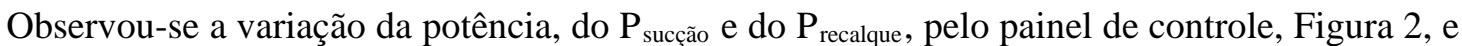
registrou-se os valores obtidos nas Tabelas 1 e 2 presentes no tópico de resultados e discussão. $\mathrm{O}$ experimento foi realizado com as velocidades médias de rotação do rotor da bomba (rev/min) de 2700 e 3000 .

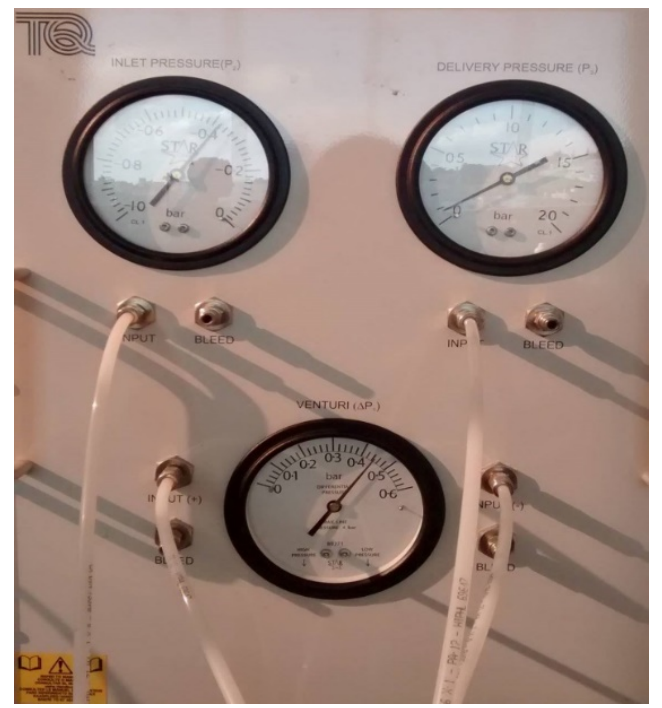

Figura 2 - Painel de controle, das variáveis $P_{\text {sucção, }} P_{\text {recalque }}$ e $\Delta P$. Figura 3.

O Tubo de Venturi que foi utilizado no experimento seguiu as dimensões apresentadas na 


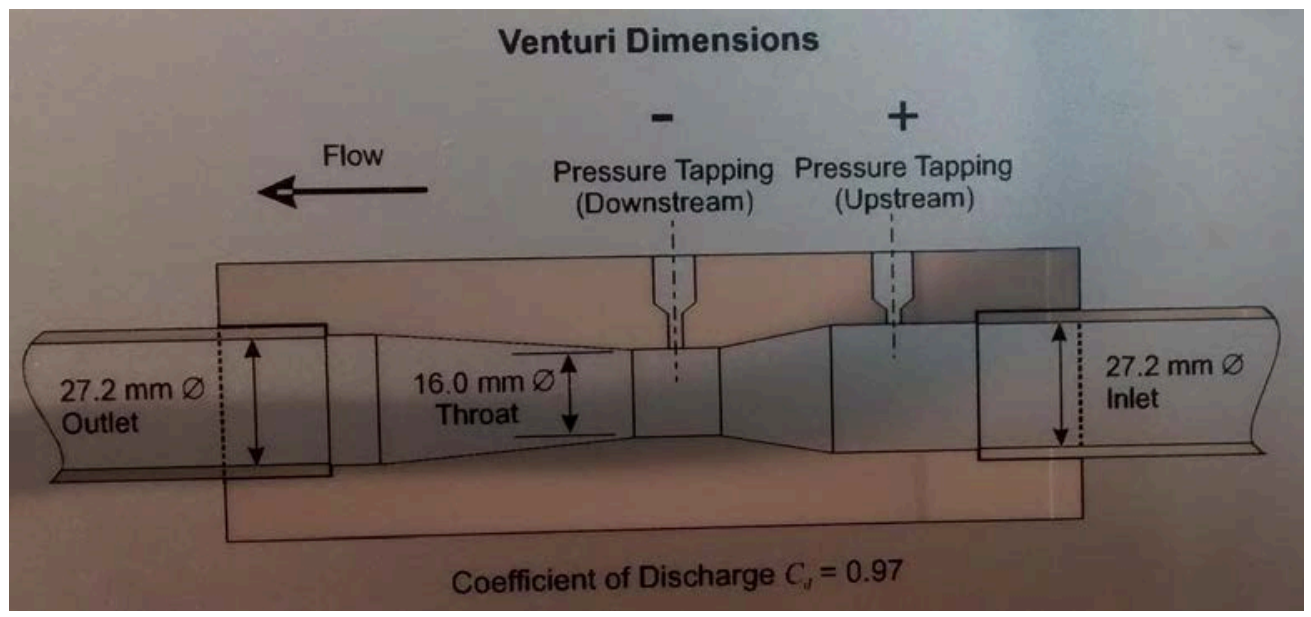

Figura 3 - Dimensões do tubo de Venturi (Imagem presente na planta piloto para informação das dimensões do tubo utilizado no procedimento)

\section{Resultados e discussão}

Os resultados obtidos com a variação do $\Delta \mathrm{P}$ venturi para as velocidades de 2700 e $3000 \mathrm{rev} / \mathrm{min}$, encontra-se nas Tabela 1 e 2 , respectivamente.

Tabela 1- Dados obtidos para a velocidade de $2700 \mathrm{ver} / \mathrm{min}$.

\begin{tabular}{lcccc}
\hline $\begin{array}{l}\text { Velocidade } \\
\text { (ver/min) }\end{array}$ & Potência $(\mathrm{W})$ & $\mathrm{P}_{\text {Suç̧ão }}(\mathrm{Pa})$ & $\begin{array}{c}\mathrm{P}_{\text {Recalque }} \\
(\mathrm{Pa})\end{array}$ & $\Delta \mathrm{P}(\mathrm{Pa})$ \\
\hline 2701 & 246 & $-0,38$ & 0,10 & 0,44 \\
2703 & 243 & $-0,35$ & 0,20 & 0,40 \\
2705 & 239 & $-0,32$ & 0,32 & 0,34 \\
2709 & 236 & $-0,29$ & 0,40 & 0,30 \\
2701 & 210 & $-0,22$ & 0,60 & 0,20 \\
2701 & 183 & $-0,16$ & 0,83 & 0,10 \\
\hline
\end{tabular}

Tabela 2- Dados obtidos para a velocidade de $3000 \mathrm{ver} / \mathrm{min}$.

\begin{tabular}{lcccc}
\hline $\begin{array}{l}\text { Velocidade } \\
\text { (ver/min) }\end{array}$ & Potência $(\mathrm{W})$ & P Suç̧ão $(\mathrm{Pa})$ & $\begin{array}{c}\mathrm{P}_{\text {Recalque }} \\
(\mathrm{Pa})\end{array}$ & $\Delta \mathrm{P}(\mathrm{Pa})$ \\
\hline 3000 & 336 & $-0,42$ & 0,21 & 0,50 \\
3005 & 328 & $-0,36$ & 0,43 & 0,40 \\
3001 & 311 & $-0,29$ & 0,65 & 0,30 \\
3005 & 281 & $-0,22$ & 0,89 & 0,20 \\
3004 & 252 & $-0,20$ & 0,96 & 0,16 \\
3000 & 239 & $-0,16$ & 1,10 & 0,10 \\
\hline
\end{tabular}

Para o cálculo da vazão volumétrica é necessário conhecermos os valores das áreas das seç̧ões transversais do tubo de maior e menor diâmetro do Tubo de Venturi. Portanto, $D_{1}=0,0272 m$ e $D_{2}=0,016 m$. 
Logo,

$$
\begin{aligned}
& A_{1}=\frac{\pi D_{1}^{2}}{4}=\frac{\pi x 0,0272^{2}}{4}=0,000581 \mathrm{~m}^{2} \\
& A_{2}=\frac{\pi D_{2}^{2}}{4}=\frac{\pi x 0,016^{2}}{4}=0,000201 \mathrm{~m}^{2}
\end{aligned}
$$

Como mostrado anteriormente na Figura 3 temos que $C d$ é o coeficiente de descarga do Tubo de Venturi, este é igual a 0,97. O valor da densidade da água foi de $1000 \mathrm{~kg} / \mathrm{m}^{3}$.

A velocidade de rotação do rotor da bomba designada para o nosso grupo foi de $2700 \mathrm{rev} / \mathrm{min}$ e $3000 \mathrm{rev} / \mathrm{min}$, tentou-se ajusta-las ao máximo, mas devido à dificuldade de controle não foi possível mantê-las nestes valores. Portanto, coletamos os valores da velocidade de rotação da bomba em cada evento, como pode-se observar nas Tabelas 1 e 2, e foi feita a média aritmética entre eles para que nos cálculos usássemos um valor constante para a velocidade de rotação da bomba.

$$
\begin{aligned}
& v_{\text {bomba }}=\frac{2701+2703+2705+2709+2701+2701}{6}=2703,33 \mathrm{rev} / \mathrm{min} \\
& v_{b o m b a}=\frac{3000+3005+3001+3005+3004+3000}{6}=3002,50 \mathrm{rev} / \mathrm{min}
\end{aligned}
$$

Foram plotados os gráficos de Hproj x Q, Pbomba x Q e Efic. x Q para cada velocidade de rotação do rotor da bomba, Gráfico 1 e 2.

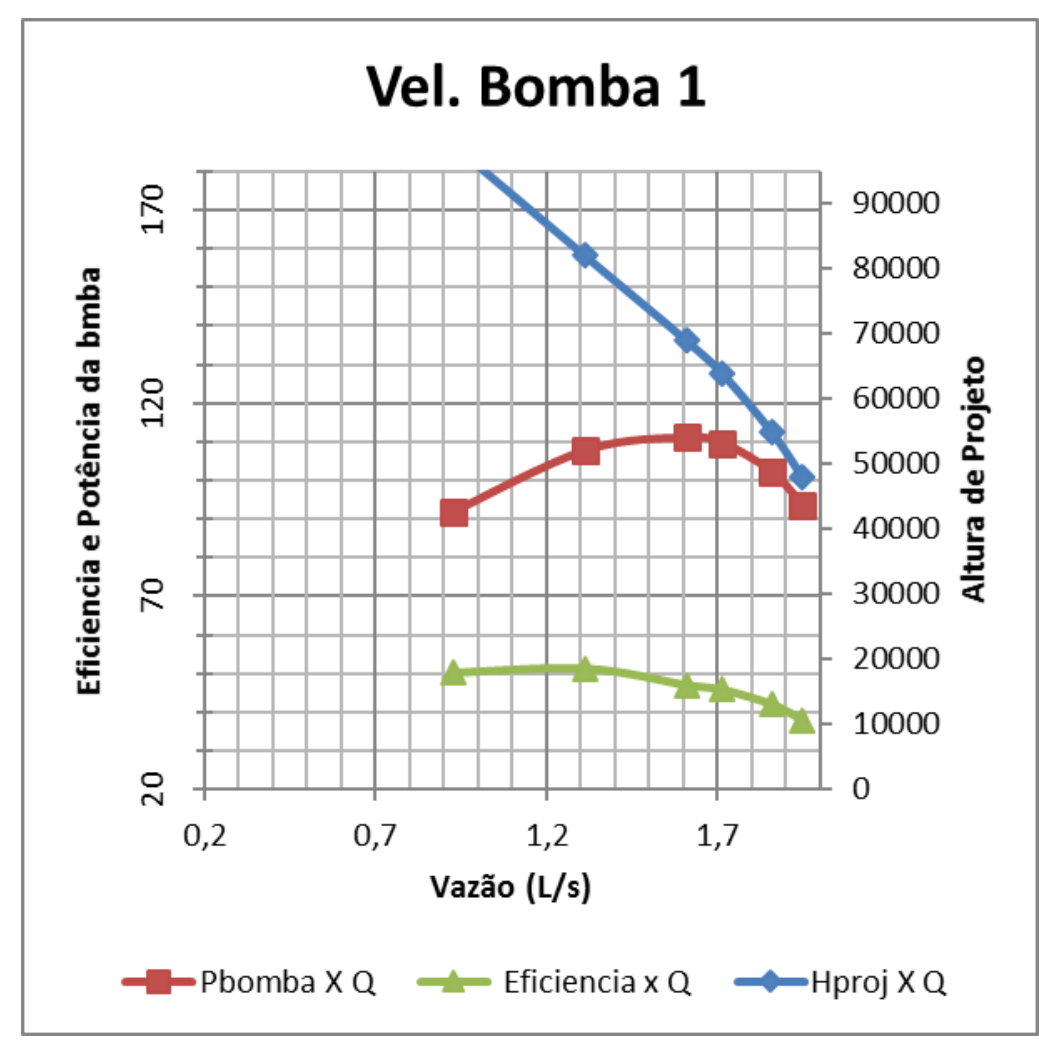

Gráfico 1 - Hproj x Q, Pbomba x Q e Efic. x Q para 2700 rev/min. 


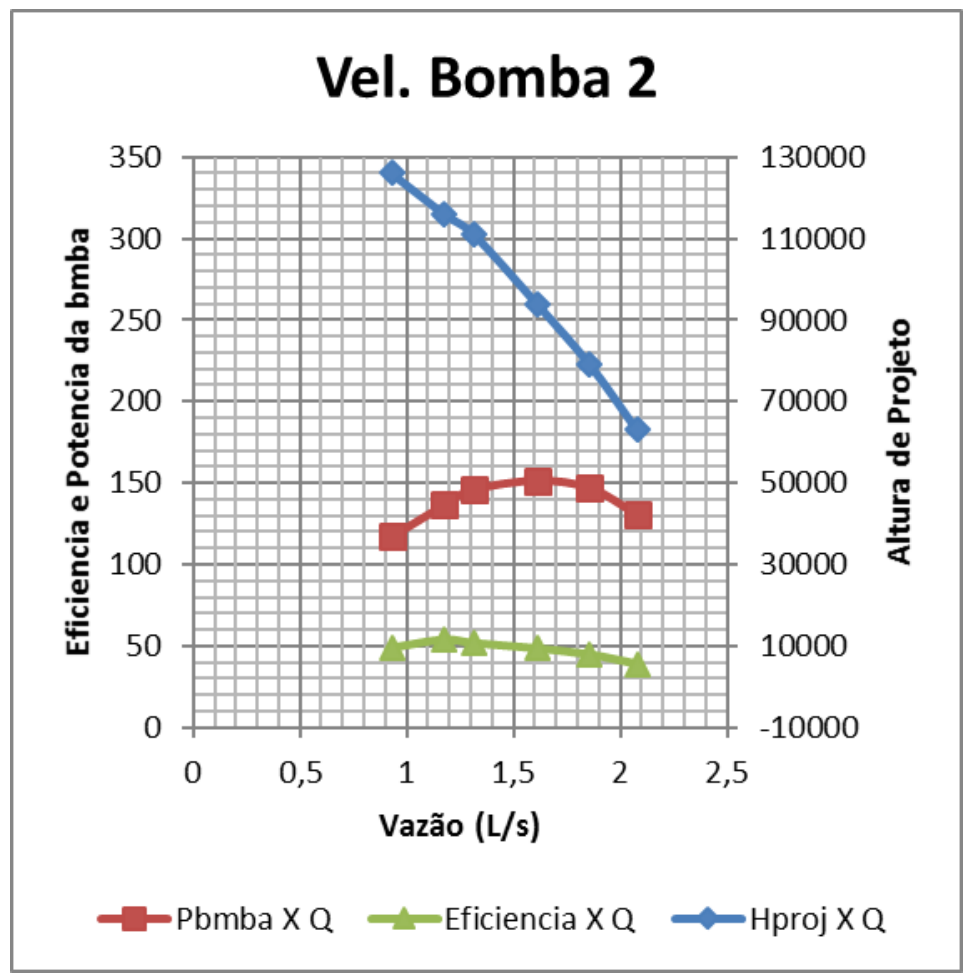

Gráfico 2 - Hproj x Q, Pbomba x Q e Efic. x Q para 3000 rev/min.

Ao realizar a comparação entre as alturas de projeto percebe-se a partir do gráfico de Hproj x Q apresentado no Gráfico 3, onde Hproj1 corresponde a curva da bomba com velocidade de 2700 (rev/min) e Hproj2 corresponde a curva da bomba com velocidade 3000 (ver/min). Podemos observar que com o aumento da velocidade de rotação da bomba maior é a Altura de Projeto correspondente a uma mesma vazão volumétrica. Dessa forma podemos dizer que a Altura de Projeto é função da velocidade de rotação da bomba e é diretamente proporcional a essa, sendo assim função da mesma. 


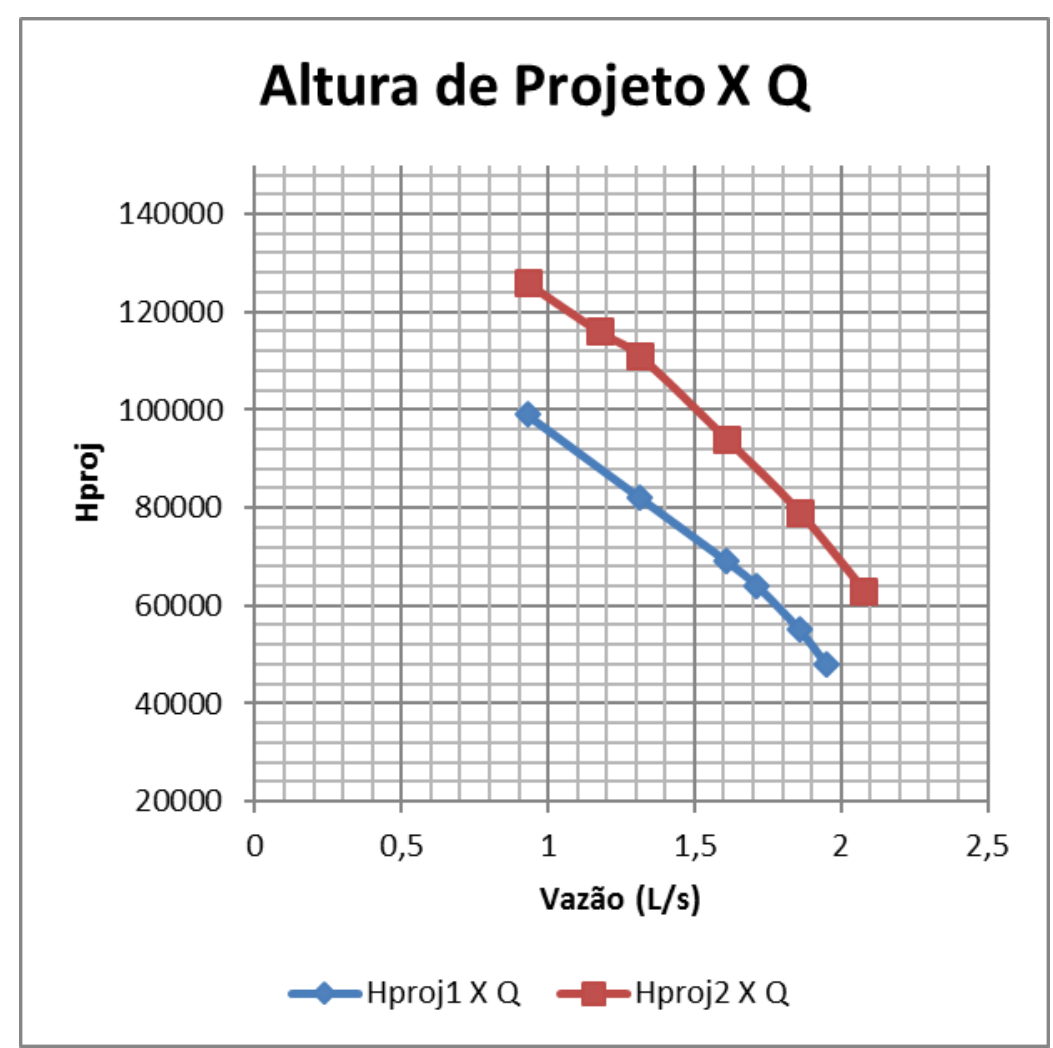

Gráfico 3 - Hproj x Q para as duas velocidades da bomba.

Ao realizar a comparação entre as potências mínimas requeridas pela bomba percebe-se a partir do gráfico de Pbomba x Q apresentado no Gráfico 4, onde Pbomba1 corresponde a curva da bomba com velocidade de 2700(rev/min) e Pbomba2 corresponde a curva da bomba com velocidade 3000 (ver/min). Podemos observar que com o aumento da velocidade de rotação da bomba maior é a Potência Mínima requerida pela bomba correspondente a uma mesma vazão volumétrica. Podemos dizer então que a Potência mínima requerida pela bomba é diretamente proporcional à velocidade de rotação da bomba. 


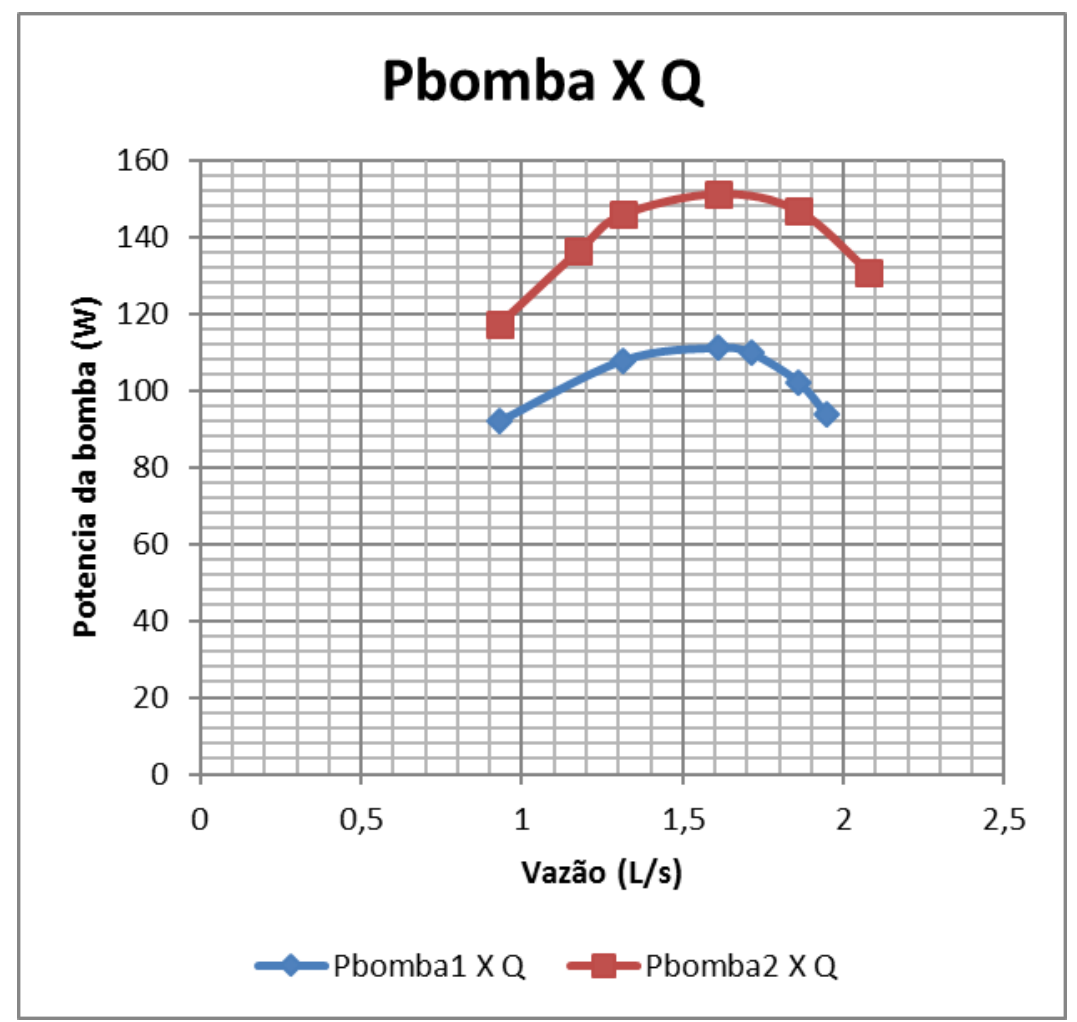

Gráfico 4 - Pbomba x Q para as duas velocidades da bomba.

Ao realizar a comparação entre as eficiências percebe-se a partir do gráfico de Eficiência x Q apresentado no Gráfico 5, onde Efic1 corresponde a curva da bomba com velocidade de 2700(rev/min) e Efic2 corresponde a curva da bomba com velocidade 3000(ver/min). Pode-se observar que, diferentemente das outras variáveis comparadas neste experimento, a eficiência permanece praticamente inalterada ao variarmos a velocidade de rotação do rotor da bomba para uma mesma vazão volumétrica. Portanto, podemos dizer que a eficiência da bomba não depende da velocidade de rotação da bomba. 


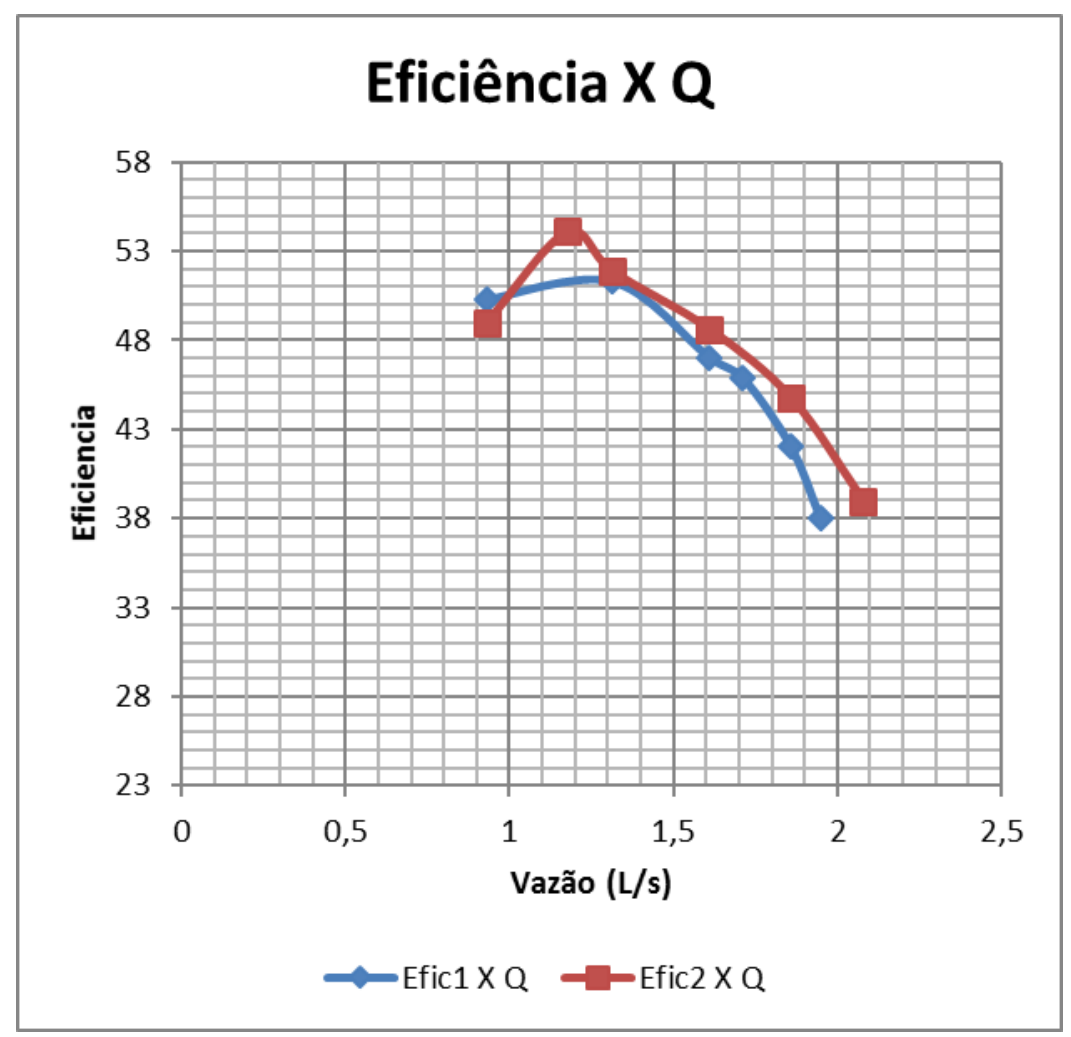

Gráfico 5 - Eficiência x Q para as duas velocidades da bomba.

\section{Conclusão}

Após análise de cada curva avaliando a influência da velocidade de rotação, mantendo uma vazão constante, sobre a carga, potência e eficiência percebeu-se que a carga (Altura de Projeto) aumenta de acordo com o aumento da vazão e com a velocidade, o que faz com que essas grandezas sejam diretamente proporcionais, o mesmo acontece com a potência da bomba, já a eficiência apresentou um comportamento similar, independente das velocidades de rotação, logo a eficiência da bomba não depende da variável velocidade.

\section{Agradecimento}

Os autores desse trabalho agradecem a Universidade Federal de Campina Grande e a Unidade Acadêmica de Engenharia Química. 


\section{Operation of a pilot plant for performance evaluation of a centrifugal pump.}

Albstract: Because of the great need of pumping system in industries and even in everyday society, it has become fundamental in-depth study of the behavior of variables related to each type of pump, when these variables compared to the variables of the system itself can determine whether the cavitation phenomenon occurs pump or not. For a given pump in a required analysis system, it is essential to evaluate the featured curves which describe the operational performance of the pumps. In this context, this study aims to evaluate the performance of a centrifugal pump with two rotational speeds, through the featured curves obtained from behavioral variables project height, pump power and pump efficiency, which was found from the Venturi tube. For this purpose, the study proposes a pilot plant which reproduces a pumping system, where the recurrent fluid in the system is water. By analyzing the data, we realized the proportionality between the project height, flow and speed variables; the pump efficiency maintained its similar behavior at all stages showing their independence in relation to the speed.

Keywords: Centrifugal pump; Project height; power; Efficiency.

\section{Referências bibliográficas}

BONNIARD, M. C. Identificação de Defeitos em Bombas de Grande Porte Através do Método de Decomposição Ortogonal de Karhunen. Tese de Mestrado em Engenharia Mecânica. Universidade Federal do Rio de Janeiro (UFRJ), 79 páginas. Rio de Janeiro, 2011.

BRIDI, A. B. Avaliação do Consumo Energético No Controle de Vazão Em Sistema de Bombeamento Utilizando Válvulas Mecânicas e Inversores de Frequência - Experiências Laboratoriais e Estudo de Caso. Tese de Mestrado em Engenharia de Edificações e Ambiental. Universidade Federal do Mato Grosso (UFMT), 153 páginas. Mato Grosso, 2013.

SOUZA, V. L. Estudo Técnico-Econômico da Substituição de Gaxetas Por Selos Mecânicos em Bombas Centrífugas. Tese de Mestrado em Engenharia Mecânica, 63 páginas. Universidade de Taubaté. São Paulo, 2009. 